\title{
Intracavitary Route of Administration
}

National Cancer Institute

\section{Source}

National Cancer Institute. Intracavitary Route of Administration. NCI Thesaurus. Code C38231.

Administration of a drug within any natural, non-pathologic cavity, such as that of cervix, uterus, or penis. 